\title{
The Panama Aging Research Initiative Longitudinal Study
}

\author{
Alcibiades E. Villarreal PhD, Ambar R. Pérez-Lao, Diana C. Oviedo PhD, Shantal Grajales MS, Maria B. Carreira PhD, \\ Gabrielle B. Britton PhD, the Panama Aging Research Initiative Group
}

\begin{abstract}
The Panama Aging Research Initiative is a cohort study of 423 adults aged $\geq 65$ years recruited from an outpatient geriatric department of Panama's largest public hospital, the Social Security Fund's Dr Arnulfo Arias Madrid Hospital Complex (Complejo Hospitalario Dr Arnulfo Arias Madrid de la Caja de Seguro Social). The study provides the first reports of modifiable and non-modifiable risk factors of cognitive impairment and dementia, as well as various health conditions common among older adults in Panama, including chronic illnesses, polypharmacy and rates of comorbidity. The initial study, conducted September 2012-May 2016, included a clinical interview; physical assessments of body mass index and handgrip strength; and cognitive testing, plus non-fasting blood draws for measurements of genetic (Apolipoprotein E genotype) and blood-based biological markers.
\end{abstract}

\section{INTRODUCTION}

Population aging projections suggest that by 2025 about threequarters of the world's population aged $\geq 60$ years will reside in developing countries.[1] This later stage of the demographic transition, resulting from declining fertility and infant mortality combined with increased life expectancy, is accompanied by significant social and economic challenges associated with caring for an increasingly older adult population. In Panama with a population of 4 million (2017), little emphasis has been placed on programs to prevent and manage risk factors for the most prevalent health problems associated with aging. In light of a growing older adult population-persons aged $\geq 60$ years are expected to reach $23.5 \%$ of Panama's population by 2050 [2] —it is urgent to address aging-related health issues.

The Panama Aging Research Initiative (PARI) is the first prospective study of age-related health factors in older Panamanians. Due to the rapid growth of Hispanic populations in the Americas and the extant literature documenting a significant impact of race and ethnicity on disease status,[3] this line of research contributes not only to national but also international aging-related research. PARI aims to (1) characterize demographic, clinical and genetic factors associated with cognitive impairment; (2) describe associated risks, including demographic, genetic and health-related factors, such as Apolipoprotein E (ApoE) genotype and noncommunicable chronic diseases; (3) examine the utility of non-

IMPORTANCE: As the first evidence of social, behavioral and biological factors related to the aging process in Panama, the study cohort of older adults provides a baseline for prospective research on cognitive impairment and biomarkers useful in early detection of dementia. Due to the worldwide trend towards aging populations and the rapid growth of Hispanic populations throughout the Americas, this line of research may provide useful results for both national and international health strategies.
Information was collected regarding limitations in activities of daily living, symptoms of depression and fall incidents. A subsample of participants provided cerebrospinal fluid to measure proteins related to Alzheimer's disease; another subsample underwent ultrasonography and electroencephalography.

This report describes the general study design and highlights lessons learned and future directions. In particular, drawing on lessons learned from this clinical research, a community-based prospective cohort study is currently under way among older adults in Panama to validate a blood-based biomarker profile for detecting mild cognitive impairment and Alzheimer's disease, as well as risk factors for cognitive decline.

KEYWORDS: Dementia, biomarkers, Latin America, aging, cognition, chronic disease, Panama

invasive techniques such as electroencephalography (EEG) and ultrasonography to determine risk of cognitive impairment; and (4) examine links between blood-based biomarkers and dementia using a longitudinal approach. To date, PARI has found various risk factors associated with dementia and cognitive impairment and provided evidence of the feasibility of applying methods to identify individuals at risk for these conditions. This report describes the general research study design, and highlights lessons learned and future directions.

\section{INTERVENTION}

The methodology published previously was based on standard protocols for conducting longitudinal studies of cognitive health in older adults.[4-8] Participants were recruited from the outpatient geriatric services of the Social Security Fund's Dr Arnulfo Arias Madrid Hospital Complex (Complejo Hospitalario Dr Arnulfo Arias Madrid de la Caja de Seguro Social, CHAAM-CSS), the largest public hospital in Panama City. Inclusion criteria were age $\geq 65$ years and willingness to participate in baseline and followup assessments. Exclusion criteria were any medical condition requiring hospitalization and/or participation in an ongoing clinical study at the time of enrollment. In total, 423 participants were enrolled from September 2012 through January 2013; followup assessments were conducted in participant subsamples from February 2013 through January 2016. Table 1 summarizes the variables and instruments employed at baseline and in followup studies. The protocol was approved by the National Bioethics Committee of the Gorgas Commemorative Institute of Health Studies (Instituto Conmemorativo Gorgas de Estudios de la Salud), the only competent national bioethics committee at that time. All participants (or their caregivers) provided written informed consent in accordance with the Declaration of Helsinki.[9]

Participant interviews and clinical exams Interviews were conducted by health professionals as well as medical, undergraduate and graduate students trained by a multidisciplinary group of specialists. Sociodemographic data collected included age, sex, years of education completed, marital status and household income. Chronic conditions, medications and performance in basic 
Table 1 Variables and instruments by study phase

\begin{tabular}{|c|c|c|}
\hline & $\begin{array}{l}\text { Base- } \\
\text { line }\end{array}$ & $\begin{array}{c}\text { Followup } \\
\text { assessments }\end{array}$ \\
\hline \multicolumn{3}{|l|}{ Sociodemographic factor } \\
\hline $\begin{array}{l}\text { Age, sex, marital status, educational level } \\
\text { completed }\end{array}$ & $x$ & $x$ \\
\hline Household income & $\mathrm{X}$ & \\
\hline Current or past occupation & $x$ & \\
\hline \multicolumn{3}{|l|}{ Clinical interview } \\
\hline Chronic conditions & $\mathrm{X}$ & \\
\hline Number of medications & $x$ & \\
\hline BADL and IADL limitations $[4,7]$ & $x$ & $x$ \\
\hline Tobacco use & $\mathrm{x}$ & \\
\hline Neuropsychiatric status $(n=204)$ & $x$ & \\
\hline EQ-5D-3L & & $\mathrm{X}$ \\
\hline $\mathrm{FAQ}$ & & $x$ \\
\hline Lawton-Brody IADL Scale & & $x$ \\
\hline GDS-30[11] & $x$ & $x$ \\
\hline \multicolumn{3}{|l|}{ Physical assessments } \\
\hline Weight, height, pulse/blood pressure & $\mathrm{x}$ & $x$ \\
\hline Handgrip strength $(\mathrm{kg})$ & $x$ & $\mathrm{x}$ \\
\hline Electroencephalography $(n=60)$ & & $x$ \\
\hline Ultrasonography $(n=159)$ & & $\mathrm{x}$ \\
\hline \multicolumn{3}{|l|}{ Cognitive function $[4,8]$} \\
\hline MMSE & & $x$ \\
\hline TMT $A$ and $B$ & & $x$ \\
\hline Clock drawing test: order & & $x$ \\
\hline Clock drawing test: copy & & $x$ \\
\hline Wechsler Memory Scale: Digit Span & & $x$ \\
\hline Semantic Verbal Fluency & & $x$ \\
\hline Boston Naming & & $x$ \\
\hline Phototest & & $x$ \\
\hline $\begin{array}{l}\text { Poppelreuter figure visual perceptual func- } \\
\text { tion test }\end{array}$ & & $\mathrm{x}$ \\
\hline Object use test: imitation and verbal request & & $x$ \\
\hline Verbal comprehension & & $x$ \\
\hline 10-word free recall & & $x$ \\
\hline Global Deterioration Scale & & $x$ \\
\hline \multicolumn{3}{|l|}{ Biological Assays } \\
\hline Blood chemistry & $\mathrm{X}$ & $x$ \\
\hline Apolipoprotein E genotype $(n=267)[5,6]$ & & $x$ \\
\hline $\begin{array}{l}\text { Blood based biomarkers (24-protein panel; } \\
\mathrm{n}=267)[5]\end{array}$ & & $x$ \\
\hline CSF biomarkers (T-tau, P-tau, Ab 1-42; $n=51$ ) & & $x$ \\
\hline
\end{tabular}

$A \beta$ 1-42: amyloid $\beta$ 1-42; BADL: Basic activities of daily living; COPD: Chronic obstructive pulmonary disease; CSF: Cerebrospinal fluid; EQ-5D-3L: European Quality of Life,EuroQol Questionnaire five-dimensional three-level version; FAQ: Functional Activity Questionnaire; GDS-30: Geriatric Depression Scale (30-item); IADL: Instrumental activities of daily living; MMSE: Mini Mental State Examination; P-tau: phosphorylated tau protein;TMT A and B: Trail Making Test A and B; T-tau: total tau protein.

24-protein panel: Eotaxin 3 and Adinoponectin; A2M: alfa-2 Macroglobulin; B2M: beta-2-microglobulin;CA-125: Cancer Antigen-125; CRP: C-reactive Protein; FABP: Fatty Acid-Binding Protein; FVII: Factor VII; 1309: T-Lymphocyte-Secreted Protein I-309; IL-1B: Interleukin-1 Beta; IL-5: Interleukin-5; IL-6: Interleukin-6; IL-7: Interleukin-7; IL-10: Interleukin-10; IL-18: Interleukin-18; MIP-1a: Macrophage Inflammatory Protein-1 Alpha; PPY: Pancreatic Polypeptide Y; SAA: Serum Amyloid Protein A; slCAM1: Intercellular Adhesion Molecule; sVCAM1: Vascular Cell Adhesion Molecule: TARC: Thymus and activation-regulated chemokine; THPO: Thrombopoietin; TN-C: Tenascin-C; TNF-a: Tumor Necrosis Factor alpha. and instrumental activities of daily living (BADL and IADL, respectively) were evaluated through self-report as published previously. $[4,7]$ Clinical exams measured body mass index $\left(\mathrm{BMl} ; \mathrm{kg} / \mathrm{m}^{2}\right)$, handgrip strength $(\mathrm{kg})$, pulse rate and blood pressure.

Cognitive, psychiatric and other assessments Cognitive $(\mathrm{n}=$ 328) and psychiatric $(n=204)$ assessments were conducted during the first followup screening ( $\mathrm{M}=4.5$ months after baseline, $\mathrm{SD}=$ 1.9 ), and a non-fasting blood draw was performed. Global cognition was assessed using the 30-item Spanish version of the Mini-Mental State Examination (MMSE)[10] adjusted for age and education. To screen for depressive symptoms, the Spanish version of the 30item Geriatric Depression Scale (GDS-30)[11] was administered by an interviewer. Additional health measures included current or past tobacco use and frequency of physical exercise.

A subsample of participants $(n=93)$ was evaluated with a complete cognitive test battery approximately 17 months $(\mathrm{M}=16.8$, $\mathrm{SD}=3.4$ ) after baseline evaluations. [8] The assessment included clinical and demographic factors and cognitive/functional tests, summarized in Table 1. The protocol also included a non-fasting blood draw. In this sample, diagnoses of Alzheimer's disease $(\mathrm{AD})$, mild cognitive impairment (MCI), no cognitive impairment or other, were made by a consensus committee according to published criteria.[12,13] Participants were classified as controls if they performed within normal limits in the neuropsychological assessment and scored below the threshold for symptoms of depression in the GDS-30. The Global Deterioration Scale[14] was used to provide information on the stage of illness or condition. The tests in the battery assessed the following cognitive domains: global cognition, language, visuospatial ability, memory, executive function, attention, praxis and gnosis.

EEGs were obtained in a subsample of participants $(n=60)$ selected at random using a standard 10-20 system (Natus Medical Incorporated, Canada). Records were classified as normal or abnormal by an experienced clinician blind to all other participant information. In another subsample $(n=159)$, also selected at random, high resolution B-mode ultrasonography was used to measure volume and speed of blood flow, intima media thickness (IMT), and presence of stenosis in the left carotid artery. The procedure for obtaining and classifying these measures is described in a recent report.[8]

Biological assays Serum samples were obtained from $63 \%$ of participants and assayed in duplicate via a multiplex biomarker assay platform using electrochemiluminescence. In order to pursue studies in biomarker discovery, we partnered with Dr Sid O'Bryant at the University of North Texas Health Science Center, whose team has a long history of conducting aging studies with Mexican-Americans.[15-17] Our current aim is to identify blood biomarkers that could be used to select potential clinical trial subjects, improve early diagnosis and enable longitudinal tracking of various disease indicators over extended periods of time. For comparative purposes, subjects were divided into three age groups: $65-74$ years, $75-84$ years and $\geq 85$ years.

Cerebrospinal fluid samples (CSF; 5-10 mL) were obtained from participants who provided additional written informed consent $(n=$ 51). Standardized lumbar puncture procedure was performed during morning hours by experienced professionals using a 20- or 22-gauge 
needle after local lidocaine application. Samples were centrifuged at low speed to discard any cellular material, then aliquoted into individual polypropylene tubes $(400-500 \mu \mathrm{L})$ without preservative, and frozen at $-80{ }^{\circ} \mathrm{C}$ until analysis. Protein concentrations for $A \beta 1-42$, T-tau, and P-tau181 were measured using standardized commercially available ELISA methods [INNOTEST $\beta$-AMYLOID(1-42), INNOTEST hTAU Ag and INNOTEST PHOSPHO-TAU(181P)], according to manufacturer specifications (Fujirebio Europe NV, formerly Innogenetics NV, Ghent, Belgium). Samples were run in duplicate along with the calibration controls and validation solutions.

\section{LESSONS LEARNED FROM INTERVENTION RESULTS}

Mean age of participants was 79.1 years $(S D=7.7)$. Female participants outnumbered male by a ratio of $2: 1$. Mean level of education was 7.3 years $(S D=4.4)$ and inversely related to age, with the oldest participants having the lowest levels of education. Participants, on average, were taking 3.9 medications $(S D=2.0)$ and levels of polypharmacy did not differ among age groups. As expected, hypertension was the most frequent chronic condition followed by diabetes and ischemic heart disease. In our sample, $59.7 \%$ of individuals aged $\geq 85$ years were classified as cognitively impaired, consistent with other studies in the region in individuals with low levels of education.[18] Increased age combined with low educational level were found to be primary risk factors for cognitive impairment. Among the cognitive, physical function and depression variables, participants aged $\geq 85$ years scored lower on cognitive tests, and had more BADL and IADL limitations and depressive symptoms.[4,7]

For Panama, PARI provided the first data regarding health-related factors in older adults, a line of research that has been active in most high-income countries for decades. In the initial cohort, we collected information on a wide range of variables and reported age-related impairments in cognition and everyday function, many of which were expected based on the international literature. At the same time, we established a line of research in blood-based biomarkers of $A D$, a relatively novel but rapidly growing area of global AD research. First, we demonstrated that a 21-protein panel could reliably distinguish AD individuals from healthy controls. [5] In subsequent studies analyzing biomarker data across cohorts, we showed that pooled data of a 24-protein panel of blood biomarkers to screen for $A D$ had excellent diagnostic accuracy and positive and negative predictive power.[16]

Importantly, a blood screen as a first step in the diagnostic process in primary care can significantly reduce costs that represent a major barrier in health and social services in low-resource settings such as Panama. In our current (community-based) cohort, we continue to collect blood samples periodically in order to assess if previous findings are repeated. We are also extending our analysis to include the utility of the biomarker profile to indicate risk for incident cognitive impairment. Based on our findings to date and considering the time- and cost-effectiveness of blood tests in primary care, we consider this a promising line of research.

We found that age and APOE4 are the strongest risk factors associated with $A D$ and $\mathrm{MCl}$. This is one of our most relevant results concerning cognitive impairment and other age-related conditions.[6] Notably, $32.6 \%$ of participants tested $(n=267)$ had at least one copy of APOE4. Table 2 summarizes the distribution of APOE genotypes by age group. Our published results have revealed that APOE4 is a strong predictor of $A D$ and $M C l[4]$ and underscore the potential of blood-biomarker screening to identify those at highest risk for dementia.[15] Our studies also confirm the feasibility of applying common biomarkers to clinical evaluations of cognitive impairment (APOE, CSF and blood).[5,6]

It is important to take into account other measures that could contribute to the development of minimal requirements for detecting risk in primary care (screening for depressive symptoms, ADL limitations, handgrip strength, etc.). $[4,7]$ We showed that the GDS-30 has a high negative predictive value and thus serves as a useful tool in primary care to identify individuals who should be referred for a full psychiatric evaluation.[19]

Table 2: Distribution of APOE genotype by age group
\begin{tabular}{|l|c|c|c|c|c|c|}
\hline Age group & e2/e2 & e2/e3 & e2/e4 & e $3 / \mathrm{e} 3$ & e3/e4 & e4/e4 \\
\hline $65-74$ years & $0.0 \%$ & $5.8 \%$ & $2.3 \%$ & $57.0 \%$ & $30.2 \%$ & $4.7 \%$ \\
\hline $75-84$ years & $0.9 \%$ & $6.8 \%$ & $0.9 \%$ & $61.5 \%$ & $27.4 \%$ & $2.6 \%$ \\
\hline$\geq 85$ years & $0.0 \%$ & $6.3 \%$ & $1.6 \%$ & $57.8 \%$ & $34.4 \%$ & $0.0 \%$ \\
\hline Total $(n=267)$ & $0.4 \%$ & $6.4 \%$ & $1.5 \%$ & $59.2 \%$ & $30.0 \%$ & $2.6 \%$ \\
\hline
\end{tabular}

The study has important limitations. The sample is not representative of the entire older-adult population. Specifically, because it consists of individuals who utilize public health services, results may underestimate the extent of cognitive impairment and age-related chronic conditions among older Panamanians, and comparisons of our results with others should be made with caution. In addition, due to time constraints imposed by the hospital setting in which we collected our data, we could not schedule all tests in a single visit. Consequently, we experienced significant losses to followup assessments. This was beyond our control, although with the exception of EEG tests, we were able to evaluate an acceptable number of participants in most followup tests, resulting in published reports. $[4,8]$

Building upon lessons learned in the initial cohort, we are currently conducting a longitudinal study in a community-based cohort and have been successful in enrolling participants from diverse backgrounds using a variety of strategies (social and print media). In our current protocol, we conduct the clinical interview, physical exam, fasting blood draw and cognitive testing in one visit and repeat them on a followup visit 12-18 months later, thus reducing the dropout rate significantly. Upon the advice of clinicians, we also include measures of frailty, which are highly effective in detecting patients at risk and are cost- and time-effective in primary care settings.

We also simplified our research protocol to retain cognitive and functional measures and eliminate EEG or ultrasound markers related to cognitive impairment and the collection of CSF samples. The main reason for this modification was that students could not be trained to conduct these tests, which require paid specialists.

Another important lesson learned was to provide study participants access to their cognitive test results, which has resulted in greater participant interest and retention in the study. In fact, as a result of this initial research, we concluded that all persons should have the opportunity to discuss clinical study findings with their health care providers to determine the appropriate course of action, as is done in many other countries. 


\section{IMPLICATIONS FOR RESEARCH}

\section{AND POLICY DECISION-MAKING}

One of the most common health concerns in older adults is cognitive decline. Recently published population-based studies confirm that rates of $A D$ and $\mathrm{MCl}$ in developing countries are similar to those of developed countries[20] and are expected to increase in the coming decades due to high rates of modifiable risk factors such as diabetes, obesity and illiteracy. Thus, identifying individuals with $\mathrm{MCl}$ who are more likely to develop $A D$ or other dementias is one of the primary challenges in cognitive aging research. Importantly, evidence indicates that AD-associated neuropathology is likely to appear decades before clinical symptoms,[21] suggesting that treatments that can potentially modify disease course will likely be most effective during pre-clinical disease stages. In this regard, the search for reliable biomarkers that can aid in early detection of dementia is essential for reducing the burden of cognitive impairment.

In rapidly aging countries such as Panama where more people are living with various chronic diseases-their treatment accounting for a significant portion of the country's health care expenditures-clinical studies like PARI are urgently needed to understand how modifiable and non-modifiable risk factors interact to produce common health-related conditions in older adults, such as functional disability and cognitive impairment.

Older adults commonly experience mental health conditions, yet most healthcare professionals in Panama receive no training in their assessment and treatment. Perhaps related to this, cognitive impairment is rarely diagnosed using published diagnostic criteria and in many cases is seen as a normal part of the aging process. Complicating this scenario, family members of affected individuals rarely seek formal medical services until advanced stages of impairment, which increases the burden of aging on individuals, family members and health systems.
PARI has helped to draw attention to the importance of comprehensive (cognitive, functional and psychiatric) evaluations of older adults. The proven predictive value of GDS-30 in primary care settings has already been used by the Ministry of Health to support the screening of depressive symptoms in this population. Our program has trained an average of ten research assistants annually, most of them students in social and biomedical sciences, which in turn has increased the number of students pursuing advanced degrees focused on health and aging.

PARI's collaboration with academic institutions has been indispensable to the program's day-to-day activities and to capacity building in areas directly impacting geriatric health. We have also built a strong alliance with international collaborations and the Ministry of Health, which looks to the PARI program as the first source of metadata collected from older adults in Panama. We continue to build relationships in local communities, and to increase recruitment and retention of participants, centering on characteristics such as age, socioeconomic factors, urban-rural demographics and cognitive status (healthy, asymptomatic and $\mathrm{MCl}$ ).

Our study adds to the effort to document the cognitive status of older adults in Panama and constitutes a contribution to our understanding of the risk factors of cognitive outcomes among Hispanic populations in general. Its ultimate aim is to provide a body of evidence to support the development of public policies to improve geriatric public health care and reduce the burden of aging on health systems and individuals.

\section{ACKNOWLEDGMENTS}

A.E.V., M.B.C., D.O. and G.B.B. are supported by grants from the National Secretariat of Science Technology and Innovation (SENACYT) of Panama, the National System of Research (SNI), Santa María la Antigua University and the Melo Brain Research Project, respectively. $-1 /$ -

\section{REFERENCES}

1. World Health Organization. Report of the World Health Organization. Active ageing: a policy framework [Internet]. World Health Organization: Geneva; 2002 [cited 2018 Jan 5]. 59. Available from: https://apps.who.int/iris/hand le/10665/67215

2. Aranco N, Stampini M, Ibarrarán P, Medellín N. Panorama de envejecimiento y dependencia en América Latina y el Caribe [Internet]. Washington, D.C.: Inter-American Development Bank; 2018 Jan [cited 2018 Feb 5]. 98 p. Available from: https://publications.iadb.org/en/panorama -de-envejecimiento-y-dependencia-en-america -latina-y-el-caribe. Spanish.

3. Chen C, Zissimopoulos JM. Racial and ethnic differences in trends in dementia prevalence and risk factors in the United States. Alzheimers Dement (NY). 2018 Oct 5;4:510-20.

4. Villarreal AE, Grajales S, López L, Britton GB; Panama Aging Research I. Cognitive impairment, depression, and cooccurrence of both among the elderly in Panama: differential associations with multimorbidity and functional limitations. Biomed Res Int. 2015;2015:718701.

5. Villarreal AE, O'Bryant SE, Edwards M, Grajales S, Britton GB; Panama Aging Research I. Serum-based protein profiles of Alzheimer's disease and mild cognitive impairment in elderly Hispanics. Neurodegener Dis Manag. 2016 Jun;6(3)203-13.
6. Villarreal AE, Grajales S, O'Bryant SE, Edwards M, López L, Montalvan A, et al. Characterization of Alzheimer's Disease and mild cognitive impairment in older adults in Panama. J Alzheimers Dis. 2016 Oct 4;54(3):897-901.

7. Villarreal $A E$, Grajales $S$, López L, Oviedo DC, Carreira MB, Gómez LA, et al. Limitations in activities of daily living among dementiafree older adults in Panama. Ageing Int. 2018 Jun;43(2):237-53.

8. Oviedo DC, Lezcano $H$, Pérez AR, Villarreal AE, Carreira MB, Isaza B, et al. Vascular biomarkers and ApoE4 expression in mild cognitive impairment and Alzheimer's disease. AIMS Neurosci. 2018 Jun 16:5(2):148-61.

9. World Medical Association Declaration of Helsinki. Ethical Principles for Medical Research Involving Human Subjects. Bull World Health Organ. 2001;79(4): 373-4.

10. Folstein MF, Folstein SE, McHugh PR. "Minimental state". A practical method for grading the cognitive state of patients for the clinician. J Psychiatr Res. 1975 Nov;12(3):189-98.

11. Yesavage JA, Brink TL, Rose TL, Lum $O$, Huang V, Adey $M$, et al. Development and validation of a geriatric depression screening scale: a preliminary report. J Psychiatr Res. 1982;17(1):37-49.

12. McKhann G, Drachman D, Folstein M, Katzman $R$, Price D, Stadlan EM. Clinical diagnosis of
Alzheimer's disease: report of the NINCDSADRDA Work Group under the auspices of Department of Health and Human Services Task Force on Alzheimer's Disease. Neurology. 1984 Jul;34(7):939-44.

13. Petersen RC, Negash S. Mild cognitive impairment: an overview. CNS Spectr. 2008 Jan;13(1):45-53.

14. Reisberg B, Ferris SH, de Leon MJ, Crook T. The Global Deterioration Scale for assessment of primary degenerative dementia. Am J Psychiatry. 1982 Sep:139(9):1136-9.

15. O'Bryant SE, Xiao G, Zhang F, Edwards M, German DC, Yin X, et al. Validation of a serum screen for Alzheimer's disease across assay platforms, species, and tissues. J Alzheimers Dis. 2014;42(4):1325-35.

16. O'Bryant SE, Edwards M, Johnson L, Hall J, Villarreal $A E$, Britton $G B$, et al. A blood screening test for Alzheimer's disease. Alzheimers Dement (Amst). 2016 Jun 25;3:83-90.

17. O'Bryant SE, Xiao G, Barber R, Reisch J, Doody $R$, Fairchild $T$, et al. A serum protein-based algorithm for the detection of Alzheimer disease. Arch Neurol. 2010 Sep;67(9):1077-81.

18. Arizaga RL, Gogorza RE, Allegri RF, Baumann $\mathrm{PD}$, Morales MC, Harris P, et al. Cognitive impairment and risk factor prevalence in a population over 60 in Argentina. Dement Neuropsychol. 2014 Oct-Dec;8(4):364-70. 
19. Lezcano H, Villareal $\mathrm{AE}$, Grajales $\mathrm{S}$, Montalván $A$, Vásquez $V$, Britton $G B$. Utility of the geriatric depression scale in older adults attending the geriatric service of the Caja de Seguro Social. Rev Méd Cient. 2017;30:4-10.

20. Prince M, Acosta D, Ferri CP, Guerra M, Huang Y, Rodríguez JJ, et al. Dementia incidence and mortality in middle-income countries, and associations with indicators of cognitive reserve: a 10/66 Dementia Research Group population-based cohort study. Lancet. 2012 Jul 7:380(9836):50-8.

21. Blennow K, de León MJ, Zetterberg H. Alzheimer's disease. Lancet. 2006 Jul 29;368(9533): 387-403.

\section{THE AUTHORS}

Alcibiades E. Villarreal, biotechnologist with a doctorate in biotechnology. Researcher at Institute for Scientific Research and High-Technology Services (Instituto de Investigaciones Científicas y Servicios de Alta Tecnología, INDICASAT AIP), Panama City, Panama.

Ambar R. Pérez-Lao, project manager and psychologist with a bachelor of science degree in psychology, INDICASAT AIP, Panama City, Panama.

Diana C. Oviedo, neuropsychologist with a doctorate in neuroscience. Researcher and professor of psychology at Universidad Santa María la Antigua, and associate researcher at INDICASAT AIP, Panama City, Panama.

Shantal Grajales, biomedical engineer with a master's degree in biomedical engineering. Former Panama Aging Research Initiative (PARI) project manager.

María B. Carreira, neuroscientist with a doctorate in neuroscience, researcher at INDICASAT AIP, Panama City, Panama.

Gabrielle B. Britton, (corresponding author: gbritton@indicasat.org.pa) neuroscientist with a doctorate in psychology and neuroscience, coordinator of the Center for Neuroscience and principal investigator in PARI at INDICASAT AIP, Panama City, Panama.

\section{GROUP AUTHORSHIP:}

\section{PANAMA AGING RESEARCH}

\section{INITIATIVE}

Alejandro Vernaza MS, medical technologist in the Transplant Department at the Social Security Fund's Dr Arnulfo Arias Madrid Hospital Com- plex (CHAAM-CSS), Panama City, Panama.

Aquiles Aguilar MD, physician in the Geriatrics Department, CHAAM-CSS.

Aron Benzadon MD, physician in the Neurology Department, CHAAM-CSS.

Astevia Montalván MD, physician in the Psychiatry Department, CHAAM-CSS.

Baltazar Isaza MD, physician in the Radiology Department, CHAAM-CSS.

David Dondis MD, physician in the Neurology Department, CHAAM-CSS.

Elena Blake, university-trained medical technologist in the Transplant Department, CHAAM-CSS.

Frank Ferro MD, physician in the Geriatrics Department, CHAAM-CSS.

Gabriela Carrillo-Pujol MD, physician in the Neurophysiology Clinic.

Ivonne Torres-Atencio $\mathrm{PhD}$, researcher in the Pharmacology Department, University of Panama, Panama.

José A. Cedeño, university-trained medical technologist in the Genetics Department, CHAAM-CSS.

Josefina Fletcher MD, physician in the Neurology Department, CHAAM-CSS.

Josué Morales MD, physician in the Geriatrics Department, CHAAM-CSS.

Juan Moscoso MS, medical technologist in the Transplant Department, CHAAM-CSS.

Lavinia Wesley MD, physician in the Radiology Department, CHAAM-CSS.

Lee Anne Gómez MD, physician in the Geriatrics Department, CHAAM-CSS.

Lineth López MD, physician in the Hematology Department, CHAAM-CSS.

Luis Lee MD, physician in the Geriatrics Department, CHAAM-CSS.

Luis Ortiz MS, medical technologist in the Transplant Department, CHAAM-CSS.
Luis Sotillo MD, physician in the Genetics Department CHAAM-CSS.

María Mendieta MD, physician in the Geriatrics Department CHAAM-CSS.

Nelson Novarro MD, physician in the Neurology Department CHAAM-CSS.

Patricia González MD, physician in the Geriatrics Department CHAAM-CSS.

Rainier Rodríguez MD, physician in the Neurology Department, CHAAM-CSS.

Ramón Zarak MD, physician in the Geriatrics Department, CHAAM-CSS

Ribana Molino MD, physician in the Geriatrics Department, CHAAM-CSS.

Sid E. O'Bryant PhD, researcher at the University of North Texas Health Science Center, Dallas-Fort Worth, Texas, USA.

Vanesa González MD, physician in the Geriatrics Department, CHAAM-CSS.

Vanessa Castillo, university-trained medical technologist in the Genetics Department CHAAM-CSS.

Viterbo Osorio MD, physician in the Geriatrics Department, CHAAM-CSS.

Vivian Vásquez MD, physician in the Psychiatry Department, CHAAM-CSS.

Yina Gutierrez, university-trained medical technologist in the Transplant Department, CHAAMCSS

Submitted: October 15, 2018

Approved for publication: March 20, 2019

Disclosures: None 